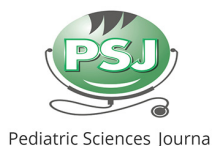

Published by Pediatrics Sciences Journal

\title{
Good outcome of newborn septic arthritis with holistic treatment: a case report
}

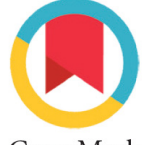

CrossMark

\author{
Muhammad Irvan Avandi i*, Brigitta Ida Resita Vebrianti Corebima ${ }^{1}$, \\ Eko Sulistijono ${ }^{1}$, Talita Clarissa Sinatra ${ }^{1}$
}

'Department of Neonatology, Child Health Division, Medical Faculty, Universitas Brawijaya, Dr. Saiful Anwar General Hospital, Malang, Indonesia

\footnotetext{
*Corresponding to:

Muhammad Irvan Avandi; Department of Neonatology, Child Health Division, Medical Faculty, Universitas Brawijaya, Dr. Saiful Anwar General Hospital,

Malang, Indonesia;

irvan avandi@yahoo.com
}

\section{Received: 2021-10-21}

Accepted: 2021-11-25

Published: 2021-12-01

\section{ABSTRACT}

Introduction: Septic arthritis (SA) is a serious joint infection associated with significant morbidity that can cause permanent damage with articular cartilage destruction, osteonecrosis, and lifelong deformities. Here we present a good outcome of newborn septic arthritis with prompt treatment.

Case Presentation: A one-month-old boy was brought with swelling of both knee joints two weeks before admission. He has a history of preterm birth and was hospitalized in the Neonatal Intensive Care Unit (NICU) because of neonatal pneumonia and sepsis. Several days after treatment, he came with swollen on both knees, both knee joint synovial liquid and sputum culture showed Klebsiella pneumoniae. Magnetic Resonance Imaging (MRI) was found intra articular fluid and erosion of the epiphyseal cartilage suspect infection process. After establishing the diagnosis of SA, we initiated treatment with cloxacillin $60 \mathrm{mg} / \mathrm{kgs}$ body weight for 14 days. He underwent debridement and physiotherapist. One year after discharge, the physical examinations showed normal findings and had a normal gait.

Conclusion: It can be concluded that prompt diagnosis and treatment of $S A$ on the newborn will have a good outcome with no sequelae. An early diagnosis of SA can guide clinicians to initiate a standardized treatment and improve patient outcomes.

Keywords: Septic Arthritis, Newborn, Preterm, Treatment.

Cite This Article: Avandi, M.I., Corebima, B.I.R.V., Sulistijono, E., Sinatra, T.C. 2021. Good outcome of newborn septic arthritis with holistic treatment: a case report. Pediatrics Sciences Journal 2(2): 29-33.

\section{INTRODUCTION}

The global incidence of septic arthritis (SA) in newborns is approximately 0.3 per 1000 live births. The incidence of SA in children younger than three months of age varies in different regions. reported an incidence of 1/1500 arthritis complicated with osteomyelitis. It was also reported that in neonates with septic arthritis and osteomyelitis, almost $70-80 \%$ were involved in lower extremities. ${ }^{1}$

Septic arthritis (SA) in newborns is a serious joint infection associated with significant morbidity that can cause permanent damage with cartilage destruction, osteonecrosis, and lifelong deformities if not diagnosed and treated promptly. Septic arthritis frequently occurs with osteomyelitis during the neonatal period. ${ }^{2}$ Septic arthritis is an inflammatory process generated by a bacterial or fungal infection. The most common etiology of the infection are Staphylococcus aureus, Haemophilus influenzae type b (HIB), and Klebsiella pneumoniae. $^{3-5}$

Prematurity, low birth infant weight, asphyxia, anemia, bacteremia, and intravenous or umbilical catheter are the leading risk factors in neonatal septic arthritis. ${ }^{3,6,7}$ The vagueness of signs and symptoms make septic arthritis difficult to diagnose. Until now, a purulent fluid joint is accepted as a gold standard to detect microorganisms in gram staining and definitive diagnosis of SA. Holistic treatment for septic arthritis children is an empirical antibiotic, surgical (arthrotomy), and other supportive treatment. ${ }^{8}$

Due to the low rate of incidence and not specific signs and symptoms in neonates, there are still difficulties diagnosing and managing it early. Early diagnosis and proper treatment are essential to obtain good outcomes and avoid sequelae. Here, we present the case of an infant with septic arthritis, which highlighted the importance of holistic treatment of SA of newborns for a better outcome.

\section{CASE PRESENTATION}

A 1-month-old baby boy was presented to the emergency room with the following chief complaint: swelling of both knee joints since two weeks before admission, the baby cried when swollen knees were touched (Figure 1).

He was admitted to the other hospital three days before admission because of a 1-week history of high fever and paleness. Laboratory results from previous hospitals indicate severe anemia and leukocytosis, and the hemoglobin was 6.3 and white blood cell 27.650. He also got a blood transfusion and injection of meropenem antibiotic from the previous admission. He was born via cesarean section at 3436 gestational age, but he did not cry immediately after birth. After birth, the baby was admitted to the Neonatal Intensive Care Unit (NICU) for three weeks because of neonatal pneumonia and sepsis. He got CPAP support and an empirical antibiotic in NICU. 

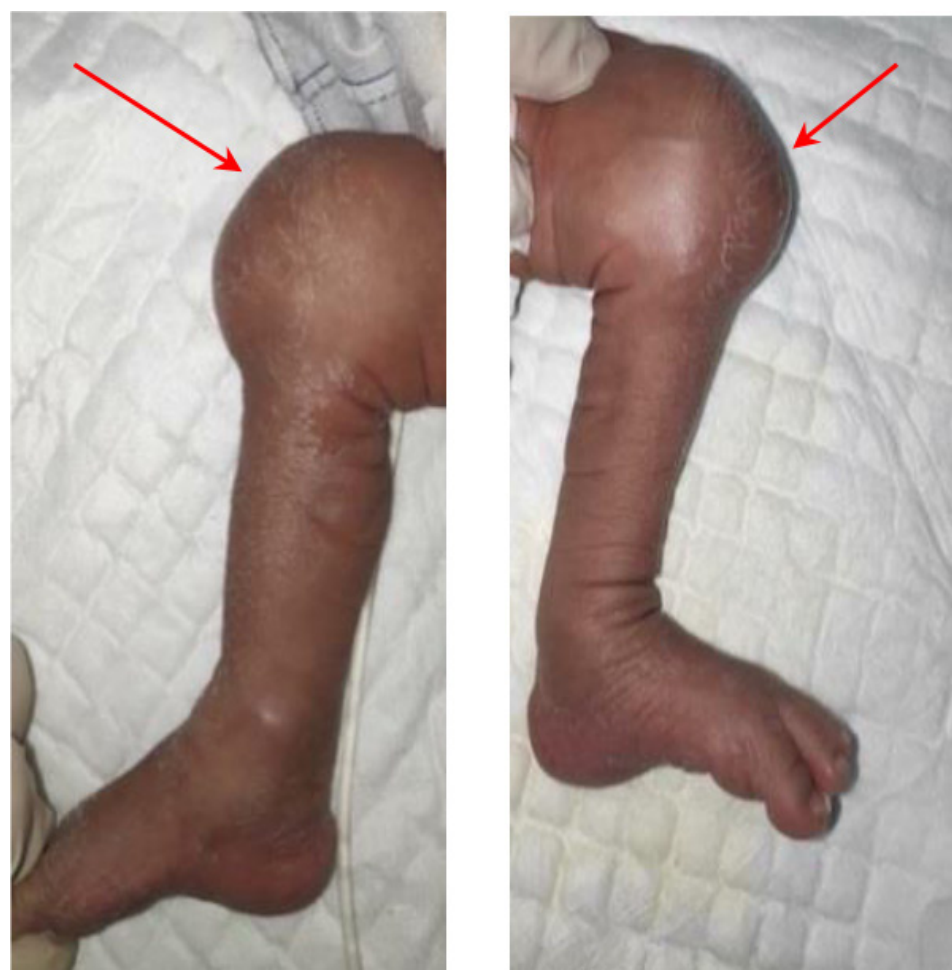

Figure 1. Bilateral knee joints

Both knees were swollen and red. In palpation, the knees were warm, tender, and painful. There were limitations in the range of motion of both knees.

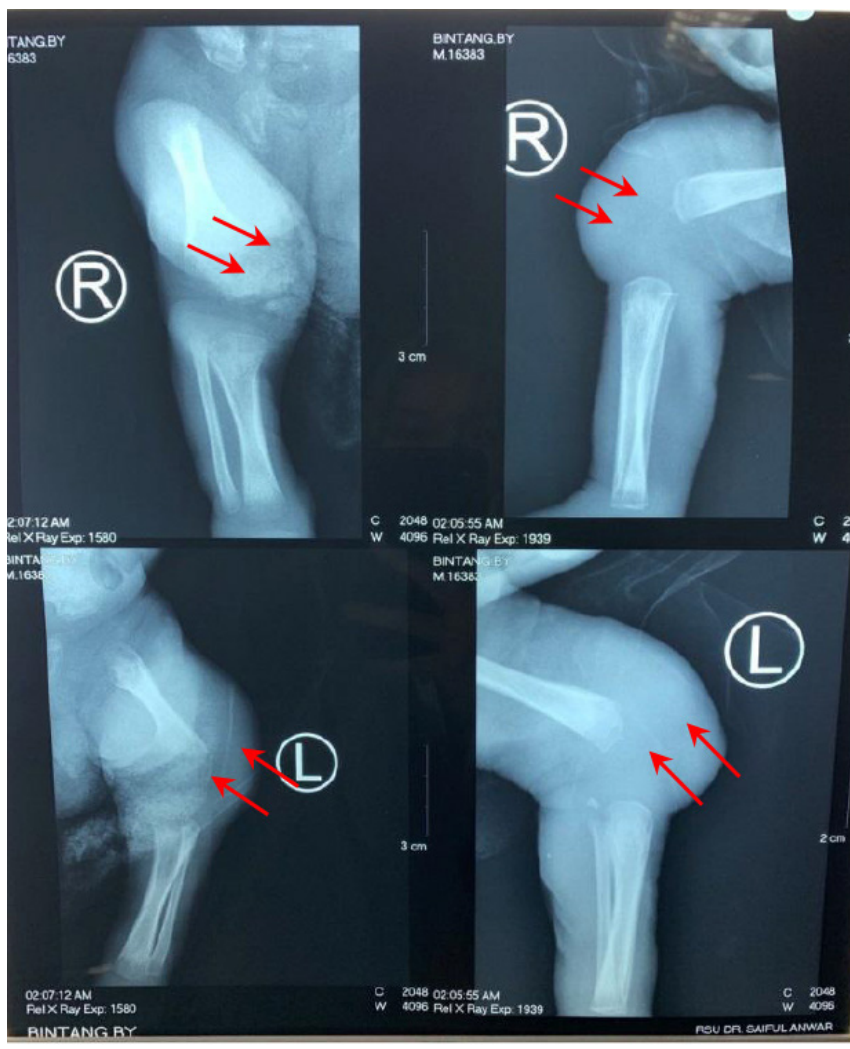

Figure 2. Bilateral knee x-ray in the first admission. Red arrows: $\mathrm{x}$-ray shows soft tissue swelling on both knees.
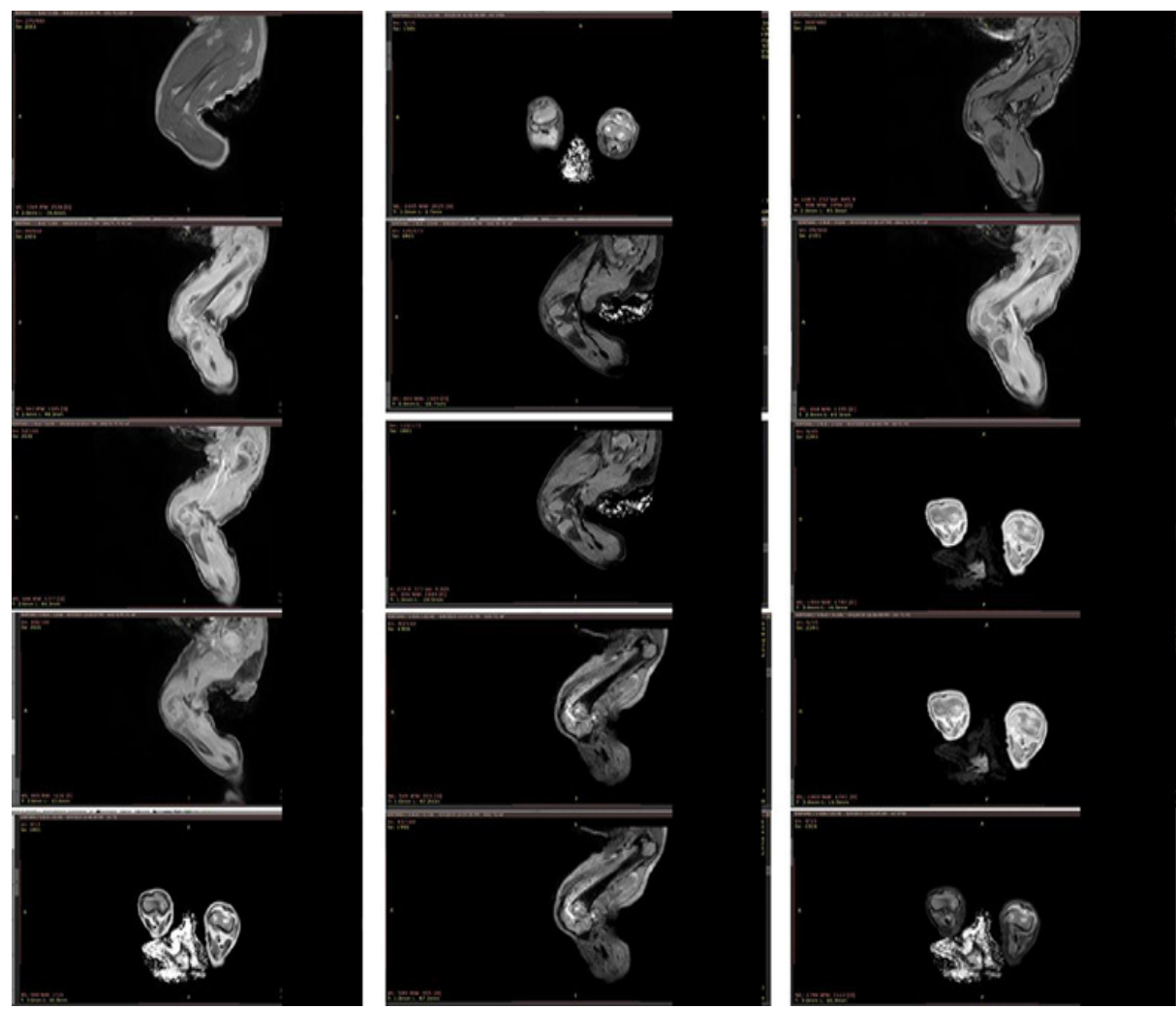

Figure 3. MRI examination result of patient's genu
Physical examination at the emergency room shows normal results, except his knees had swollen. The anthropometric measurement showed that his nutritional status was normal, his body weight was 3200 grams, his body height was $51 \mathrm{cms}$, and his head circumference was $35 \mathrm{cms}$. A complete blood count taken when he was admitted to the hospital showed hemoglobin level $10.30 \mathrm{~g} / \mathrm{dL}$; leukocyte $22.860 \times 10^{3} / \mu \mathrm{L}$; hematocrit $30.90 \%$; and thrombocyte $387.000 / \mu \mathrm{L}$. White blood cells were showed a shift to the left with neutrophil dominance $61.6 \%$ and CRP 13.72. Both knee synovial joint liquid and sputum culture showed Klebsiella pneumoniae and blood culture showed Staphylococcus hominis.

$\mathrm{X}$-ray finding was found soft tissue swelling on both knees (Figure.2), and ultrasonography examination was found fluid collection complex suprapatellar bilateral (right volume is $4 \mathrm{cc}$, left volume is $1.4 \mathrm{cc}$ ) suggest with septic arthritis with suspected abscess formation. Knee MRI was performed and found free intra articular fluid, suprapatellar pouch with 
bleeding component, and erosion of the epiphysis cartilage accompanied by warming of the bilateral (dominant left) genu suspect et causa infection process and hemophilic arthropathy (Figure.3).

During admission, the baby was treated with cloxacillin $60 \mathrm{mg} / \mathrm{kgs}$ bodyweight for 14 days and debridement was performed on both knees. He was also consulted by the physiotherapist to perform an active assistive exercise. After several days of treatment, he was discharged and followed up in the outpatient department. One year after discharge, the physical examinations showed normal findings and had a normal gait.

\section{DISCUSSION}

Septic arthritis is defined as the inflammation of the synovial membrane with purulent effusion into the joint capsule due to suppurative infection of a joint. ${ }^{9}$ The early clinical manifestations of neonatal septic arthritis are often nonspecific with signs of septicemia, painful legs, pseudo-paralysis, lack of movement, restlessness and refusal to eat or drink. Other manifestations can also be fever, neurological manifestations, respiratory distress, abdominal distension, cholestasis or irritability. ${ }^{6}$

There are fewer clinical signs of neonatal septic arthritis compared with older children and adults, making it more difficult to diagnose osteomyelitis and/ or arthritis in neonates. This condition may be caused by the newborn's immune system not being optimally developed, so they are less able to increase the immune response. Atypical clinical manifestations and generally normal laboratory results also cause diagnosis to be often delayed and may be difficult to establish., ${ }^{40}$ One of the hallmarks of septic arthritis is low glucose levels and elevated levels of protein or lactic acid in synovial fluid. However, these values are nonspecific and can be found in all forms of inflammatory arthritis. ${ }^{9,11}$ An increase in inflammatory markers such as erythrocyte sedimentation rate and C-reactive protein with leukocytosis, together with clinical history findings suggestive of septic arthritis and an increased synovial WBC count greater than $50,000 / \mu \mathrm{L}$ does have a high sensitivity. ${ }^{12,13}$ However, elevated inflammatory markers are not specific for the diagnosis of septic arthritis. ${ }^{11}$ In this case, the patient came with swelling of both knee joints two weeks before admission. No trauma was mentioned by the mother. The patient also had a fever three days before admission. There wasn't another abnormal manifestation during physical examination, the most prominent presentation was diffuse swelling of both knees accompanied by fever. The laboratory result shows an increase in PMN and CRP in blood.

Septic arthritis neonates have several imaging modalities for investigation, including plain radiography, ultrasonography, bone scintigraphy, and MRI, none of which is without shortcoming. ${ }^{3,14}$ Although plain radiographs of bone are often normal, $\mathrm{x}$-ray images can show capsule swelling. Widening of the joint space, subluxation or dislocation, radiolucency of the proximal femoral metaphysis, or periosteal elevation of the upper end of the bone. ${ }^{15}$ Ultrasonography has several advantages over bone scintigraphy and MRI; the examination process is fast, non-invasive, portable, and relatively inexpensive. Ultrasonography has been shown to confirm or exclude effusion in children safely and may also be used to identify periosteal elevation, cortical erosion, or a subperiosteal collection. ${ }^{3}$

MRI is one of the supporting examinations that can determine the extent of soft tissue involvement (multiplanar). MRI provides soft tissue and bone contrast which is particularly useful in neonates and young children because it allows assessment of cartilage (including growth plate) involvement. This examination is good for evaluating complex sites such as the spine (discitis) to exclude extradural collections causing spinal cord compression. Under active inflammation and infection conditions, there will be a low signal on T1 weighting and a high signal on T2 weighting and STIR sequences. In addition, MRI is also useful for detecting fluid in the bone and adjacent osteomyelitis. There are drawbacks to MRI, which are expensive and require sedation or general anesthesia in the neonate. ${ }^{14}$ In this patient, genu X-Ray and USG of genu have been performed, giving well visualization. Although good visualization, MRI is still needed to know the lesion of bone further and the bone infection spread. Another measure must be taken if another bone is suspected of becoming infected because the limb was threatened.

The most common bacterium that causes septic arthritis worldwide is Staphylococcus aureus. Other organisms that can also cause are Klebsiella pneumoniae, Group B streptococci, Escherichia coli, Enterobacter sp., and Candida sp. ${ }^{5}$ After entering the joint space, the bacteria will trigger an acute inflammatory process with rapid development. This condition causes a response from the synovium resulting in hyperplasia of the proliferative layer cells and the entry of inflammatory cells. In infected joints, phagocytic cells, including neutrophils and macrophages, migrate chemotactically, facilitated by a gradient of bacterial products and immune response mediators. In inflammatory conditions, neutrophils play a major role in the first line of defense against pathogens, including bacteria. Leukocytes are the first to migrate to the site of infection, while activated macrophages are recruited to the joint, followed by T-lymphocytes. This destruction is caused by inflammatory cytokines and proteases as well as microbial components. Adhesins are microbial surface components that mediate staphylococcal attachment to intra-articular proteins (e.g., elastin, laminin, fibronectin) that have been shown to influence the ability of S. aureus to cause septic arthritis. ${ }^{16}$ Theoretically, infection can reach bone (or joint) via three main routes of blood flow such as hematogenous spread, direct contamination (e.g., penetrating or stab wounds, surgery) and contamination from nearby infections (e.g., cellulitis). Nevertheless, in the neonatal group, the presence of rich vascular supply and lack of synovial basement membrane in the joints of newborn infants become predisposing factors of secondary hematogenous seeding of infective organisms in septic arthritis. $^{15}$

In this patient, the aspiration of pus culture showed Klebsiella pneumoniae with ESBL spectrum, which is one of the 
commonest bacteria found in the infection. There is no source of infection found in the child, so the infection is suspected to be late-onset sepsis via hematogenous spread. The pathology anatomy result shows many mononuclear cells reside in synovial fluid and pus suggest chronic infection. Moreover, MRI also shows a wide bleeding area that suggests a hematogenous spread source. On the other hand, the evidence of perinatal infection is supported by a history of fever during the first trimester, vaginal discharge since early pregnancy, and betel leaf herbs at the beginning of pregnancy. Other risk factors of septic arthritis in this patient include premature birth and perinatal asphyxia, resulting in transient bacteremia. The history of patient was treated in the Neonatal Intensive Care Unit (NICU) also indicates that he had multiple potential portals for entry of bacteria into the bloodstream, including indwelling lines, repeated laboratory draws, and thin skin that may be injured easily

The treatment of SA should be started without delay after synovial fluid and blood samples have been obtained with high-dose empirical IV antibiotics. At present, there is still no consensus on antibiotics therapy in septic arthritis of neonates. Several studies recommend that empirical antibiotic treatment should be initiated immediately, and later modified once the causative microorganism has been isolated. The choice of an empirical antimicrobial therapy should consider patient age, immunization status and condition (underlying disease, disease severity, and risk factors), local epidemiological patterns, the antibiotic susceptibility of local isolates, gram stain result of the aspirate, and regional microbiological profile, including the prevalence of MRSA in the community. Being the most common cause of SA, an antibiotic against Staphylococcus aureus or MRSA should always be included. ${ }^{9,12,15}$ The empiric treatment for children three months or older should cover Staphylococcus aureus and other Gram-positive organisms. The first choice is anti-staphylococcal penicillin or a first-, second-, or third-generation cephalosporin depending on the severity of the illness. ${ }^{9}$ The two studies state that the initial antibiotic therapy should cover Gram-positives (particularly S. aureus and GAS) and that clindamycin effectively treats infections due to clindamycinsensitive MRSA. ${ }^{12,15}$ When the MRSA prevalence for a specific region is $>10$ $15 \%$, then clindamycin should be added. If local clindamycin resistance rates are $>10-25 \%$, or in a setting of clindamycin inducible resistance, vancomycin should be preferred. Additional antibiotics should be considered in children not immunized against $\mathrm{Hib}^{14}$

Some infection societies and specialists have recommended 4 to 6 weeks of antibiotics by intravenous. ${ }^{11}$ However, most studies pertain to osteomyelitis and septic arthritis in neonates have reported shorter intravenous regimens (from approximately $1-4$ weeks). ${ }^{11,17}$ The empiric antibiotic regimen in infants (1 to 3 months) is combined with vancomycin and gentamicin. A $15 \mathrm{mg} / \mathrm{kg}$ dose of vancomycin is administered intravenously every 6 to 8 hours, with a target trough of 15 to $20 \mathrm{mcg} / \mathrm{ml}$. Creatinine levels and trough targets must be monitored every 2 to 3 days. A $2 \mathrm{mg} / \mathrm{kg}$ dose of gentamicin is administered intravenously every 8 hours for full-term infants. Suppose the patient is aged $<29$ weeks, a $4 \mathrm{mg} / \mathrm{kg}$ dose of gentamicin should be administered every 36 hours. If the patient is aged $\geq 29$ weeks, the same dosage should be administered every 24 hours. ${ }^{17}$ These recommendations vary markedly by institutions based on the local microbiology and epidemiology. In this patient, the antibiotic was given within two weeks and there is an improvement which suggests antibiotic treatment vary among the patient and from the clinical course.

To date, the use of systemic corticosteroids together with antibiotics in septic arthritis in pediatric patients is beneficial, although there is insufficient research evidence to support this effect in adults. One meta-analysis study demonstrated a beneficial effect of corticosteroid use in SA. It was explained that the impact of using corticosteroids as adjunctive therapy to antibiotic therapy in the treatment of pediatric patients with AS could shorten the duration of intravenous antibiotic treatment, accelerate the transition from treatment to oral antibiotics and normalization of C-reactive protein, which ultimately results in a decrease in the length of hospital stay. Therefore, it is recommended the use of corticosteroids as adjunctive therapy with antibiotics in the treatment of children with SA. ${ }^{18}$ Pathogenesis, the damage inflicted on infected joints is the result of the host inflammatory response as opposed to direct damage caused by the organism, so corticosteroids can given to suppress the inflammatory process. ${ }^{19}$ No systemic corticosteroid was given in this case, but a favorable outcome is expected.

Septic arthritis is a clinical emergency, and prevention of significant long-term morbidity depends on timely diagnosis and relief of pressure; either by ultrasoundguided joint aspiration or more invasive surgical techniques. ${ }^{20}$ Irrigation and debridement must be urgently performed to clear the joints of bacteria, associated enzymes, and degradation materials that can destroy the articular cartilage, reduce intraarticular pressure, and decrease epiphyseal ischemia. This can be done openly via an anterior approach, providing direct access to the joint and avoiding the ascending branch of the medial circumflex artery. ${ }^{21}$ The joints of neonates are small; the knee of one, for instance, is only about the size of the interphalangeal joint of an adult thumb. Therefore the incision for arthrotomy of the knee must necessarily be small. Access to the joint is consequently technically difficult and limited. Introducing and maintaining a suction drain in such small joints is also difficult. After an arthrotomy is performed, a drain is placed and irrigation is performed to allow continued drainage of the joint postoperatively. ${ }^{21}$ In this patient, debridement and arthrotomy were performed with antibiotic cloxacillin given until maximum dose. The result was good and the patient recovered clinically and resolved its sign and symptom. The prevention of nursery infections may also help to reduce the incidence of neonatal septic arthritis.

The most serious complication of septic arthritis, especially in newborns, is avascular necrosis, leading to the partial or complete destruction of the capital femoral epiphysis, the growth plate, or both. ${ }^{1}$ Septic arthritis in the neonate gives rise to more 
serious handicaps. This can be explained by the fact that in premature infants, the ossific nucleus of the end of the bone has not yet appeared, and in addition, there is no epiphyseal plate. In these neonates, the infection begins in the vulnerable cartilage precursor of the end of the bone, causing rapid destruction with consequent joint destruction, growth arrest, and subsequent leg length discrepancy. ${ }^{20,21}$ The early orthopedic, pediatric intervention is important for the early preservation of anatomical and functional support in this patient.

\section{CONCLUSION}

Difficulties in diagnosing and managing AS in neonates are caused by unclear signs and symptoms in the early stages. Therefore early diagnosis and appropriate treatment are important to get a good outcome and avoid sequelae. Several imaging modalities can be used to assess neonatal SA, including plain radiography, ultrasonography, and MRI. SA treatment should be started without delay after synovial fluid, and blood samples have been obtained, i.e., by administering highdose empiric IV antibiotics. Empirical antibiotic treatment should be initiated immediately and subsequently modified after the isolated causative microorganism. Early pediatric orthopedic intervention is also important to achieve a good prognosis in terms of anatomical and functional conditions in these patients.

\section{FUNDING}

The authors are responsible for the study funding without the involvement of grant, scholarship and any other resource of funding.

\section{CONFLICT OF INTEREST}

The authors reports no conflicts of interest in this work.

\section{AUTHOR CONTRIBUTION}

All authors contributed equally in the writing of this article

\section{CONSENT FOR PUBLICATION}

The patient's parents had agreed and signed informed consent regarding publishing the case in an academic journal without exposing his child's identity.

\section{REFERENCES}

1. Li Y, Zhou Q, Liu Y, Chen W, Li J, Yuan Z, et al. Delayed treatment of septic arthritis in the neonate: A review of 52 cases. Medicine (Baltimore). 2016;95(51):e5682.

2. Narang A, Mukhopadhyay K, Kumar P, Bhakoo $\mathrm{O}$. Bone and joint infection in neonates. Indian J Pediatr. 1998;65:461-4.

3. Samora JB, Klingele K. Septic arthritis of the neonatal hip: acute management and late reconstruction. J Am Acad Orthop Surg. 2013;21(10):632-41.

4. Offiah AC. Acute osteomyelitis, septic arthritis and discitis: differences between neonates and older children. Eur J Radiol. 2006;60(2):221-32.

5. Deshpande PG, Wagle SU, Mehta SD, Bharucha BA, Irani SF. Neonatal osteomyelitis and septic arthritis. Indian Pediatr. 1990;27(5):453-7.

6. Sankaran G, Zacharia B, Roy A, Purayil SP. Current clinical and bacteriological profile of septic arthritis in young infants: a prospective study from a tertiary referral centre. Eur J Orthop Surg Traumatol. 2018;28(4):573-8.

7. Sreenivas T, Nataraj AR, Kumar A, Menon J. Neonatal septic arthritis in a tertiary care hospital: a descriptive study. Eur J Orthop Surg Traumatol. 2016;26(5):477-81.

8. Agarwal A, Aggarwal AN. Bone and Joint Infections in Children: Septic Arthritis. Indian J Pediatr. 2016;83(8):825-33.

9. Autore G, Bernardi L, Esposito S. Update on Acute Bone and Joint Infections in Paediatrics: A Narrative Review on the Most Recent Evidence-Based Recommendations and Appropriate Antinfective Therapy. Antibiot (Basel, Switzerland). 2020;9(8).

10. ELMeneza S. The Significance of Septic Arthritis in Neonatal Intensive Care Unit. Pediatr Neonatal Biol Open Access. 2018;3(3):20-2.

11. Gigante A, Coppa V, Marinelli M, Giampaolini N, Falcioni D, Specchia N. Acute osteomyelitis and septic arthritis in children: a systematic review of systematic reviews. Eur Rev Med Pharmacol Sci. 2019;23(2 Suppl):145-58.
12. Kang S-N, Sanghera T, Mangwani J, Paterson $\mathrm{JMH}$, Ramachandran $\mathrm{M}$. The management of septic arthritis in children: systematic review of the English language literature. J Bone Joint Surg Br. 2009;91(9):1127-33.

13. Obey MR, Minaie A, Schipper JA, Hosseinzadeh P. Pediatric septic arthritis of the knee: predictors of septic hip do not apply. J Pediatr Orthop. 2019;39:e769-72.

14. Di Pietro GM, Borzani IM, Aleo S, Bosis S, Marchisio P, Tagliabue C. Pediatric Septic Arthritis of the Knee Due to a Multi-Sensitive Streptococcus pyogenes Strain Responsive to Clindamycin-A Case Report. Vol. 8, Children (Basel, Switzerland). 2021.

15. Manz N, Krieg AH, Heininger U, Ritz N. Evaluation of the current use of imaging modalities and pathogen detection in children with acute osteomyelitis and septic arthritis. Eur J Pediatr. 2018;177(7):1071-80. Available from: https://doi.org/10.1007/s00431-018$\underline{3157-3}$

16. Rutz E, Spoerri M. Septic arthritis of the paediatric hip - A review of current diagnostic approaches and therapeutic concepts. Acta Orthop Belg. 2013;79(2):123-34.

17. Kabak S, Halici M, Akcakus M, Cetin N, Narin N. Septic arthritis in patients followedup in neonatal intensive care unit. Pediatr Int. 2002;44(6):652-7.

18. Qin Y-F, Li Z-J, Li H. Corticosteroids as adjunctive therapy with antibiotics in the treatment of children with septic arthritis: a meta-analysis. Drug Des Devel Ther. 2018;12:2277-84.

19. Fogel I, Amir J, Bar-On E, Harel L. Dexamethasone Therapy for Septic Arthritis in Children. Pediatrics. 2015;136(4):e776-82.

20. Lindegaard C, van Galen G, Aarsvold S, Berg L, Verwilghen D. Haematogenous septic arthritis, physitis and osteomyelitis in foals: A tutorial review on pathogenesis, diagnosis, treatment and prognosis. Part 2. Equine Vet Educ. 2022;34(1):37-48

21. El-Sayed AMM. Treatment of early septic arthritis of the hip in children: comparison of results of open arthrotomy versus arthroscopic drainage. J Child Orthop. 2008;2(3):229-37.

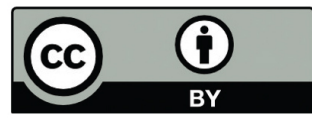

This work is licensed under a Creative Commons Attribution 